\title{
Ground state of a resonant two-qubit Rabi model in the ultrastrong coupling regime
}

\author{
Kelvin M. C. Lee and C. K. Law \\ Department of Physics and Institute of Theoretical Physics, The Chinese University of Hong Kong, \\ Shatin, Hong Kong Special Administrative Region, People's Republic of China
}

\begin{abstract}
We consider a generalized Rabi model formed by two identical qubits interacting with a common oscillator mode. In the near resonance configuration where the oscillator frequency is close to the transition frequency of the qubit, we determine the ground state of the model approximately by using a variational method and a transformation method. Both methods are shown to provide good agreement with the exact numerical ground state for a range of coupling strength in the ultrastrong regime. In addition, we indicate how the accuracy of the approximation can be further improved by using perturbation theory. We also examine the ground state entanglement between the two qubits. By calculating the negativity, we quantify the degree of entanglement as a function of coupling strength.
\end{abstract}

PACS numbers: 42.50.Pq, 03.65.Ud

\section{INTRODUCTION}

Recently, there has been a revival of interest in studying the behavior of a two-level system interacting with a quantum harmonic oscillator beyond rotating wave approximation (RWA). One of the motivations lies in the ultrastrong coupling regime recently explored by experiments with artificial atoms and cavity photon resonators [1, 2], and microcavities embedding doped quantum wells [3, 4]. In such a regime, the qubit-oscillator interacting strength is comparable to the oscillator frequency or the natural transition frequency of the qubit, and theoretical investigations have found novel phenomena, such as the asymmetry of vacuum Rabi-splitting [5], photon blockade [6], nonclassical states generation [7], superradiance transition [8], and collapse and revival dynamics [9].

For a single qubit interacting with a quantum harmonic oscillator, the system is described by the Rabi model [10] and it has been studied extensively. While there exist analytical methods [11, 12] to determine the ground state exactly, approximation schemes are often employed because the approximate ground states in their closed forms could be more physically transparent for detailed investigations 13 24.

In this paper we investigate the ground state of a generalized Rabi model in which a quantum harmonic oscillator interacts with two identical qubits symmetrically. This is a special case of the Tavis-Cummings model [25] beyond RWA. Recently Agarwal et al. [26] have employed an adiabatic approximation to determine the energy spectrum and entanglement dynamics in the quasidegenerate situation with $\omega_{A} \ll \omega_{c}$, where $\omega_{A}$ and $\omega_{c}$ are natural frequencies of the qubit and the oscillator respectively. Our work here will focus on a different situation with $\omega_{A} \approx \omega_{c}$. Such a near resonance situation is more complicated because of the break down of the adiabatic approximation.

To approach the problem, we employ two approximation schemes to determine the ground state. In Sec. II, we describe a variational solution, in which the trial ground wave function is constructed from a generalization of a single qubit problem [13, 14]. In Sec. III, a transformation method is introduced in order to simplify the Hamiltonian. Both methods turn out to give solutions that have good agreement with the exact numerical ground state. In particular the accuracy of the transformation method can be further improved by combining with perturbation theory.

An interesting feature in the system is the quantum entanglement between the two qubits. In Sec. IV, we shall characterize the entanglement by using negativity. We shall see that there exists entanglement even the system in the ground state. Such an entanglement is due to counter-rotating terms involving virtual photons. By using our approximate ground state, we quantify the entanglement by negativity as a function of the coupling strength.

\section{THE HAMILTONIAN AND VARIATIONAL GROUND STATE}

The Hamiltonian of the model is given by $(\hbar=1)$

$$
H=\omega_{A} J_{x}+\omega_{c} a^{\dagger} a+g\left(a+a^{\dagger}\right) J_{z},
$$

where $a$ and $a^{\dagger}$ are the annihilation and creation operators of the cavity field mode of frequency $\omega_{c}$. The J's are spin-1 angular momentum operators. Physically, the spin-1 system can be formed by two identical two-level atoms in the triplet space. Then $\omega_{A}$ is the atomic transition frequency, and $g$ denotes the collective atom-field coupling strength. We note that the (antisymmetric) singlet state formed by the two atoms does not couple to the field and it does not constitute the ground state of the system.

Our task in this paper is to determine the ground state energy $E_{g}$ and the ground state vector $\left|\psi_{g}\right\rangle$ of the system, which are defined by:

$$
H\left|\psi_{g}\right\rangle=E_{g}\left|\psi_{g}\right\rangle
$$

To this end, we let $|m\rangle_{A}$ be an eigenvector of $J_{z}$ (the quantum number $j=1$ is suppressed for convenience), 


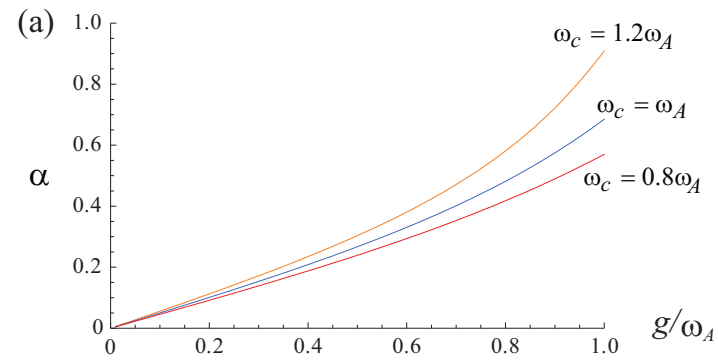

(b)

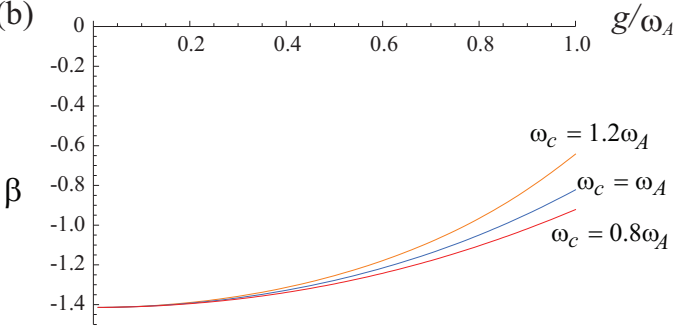

(c)

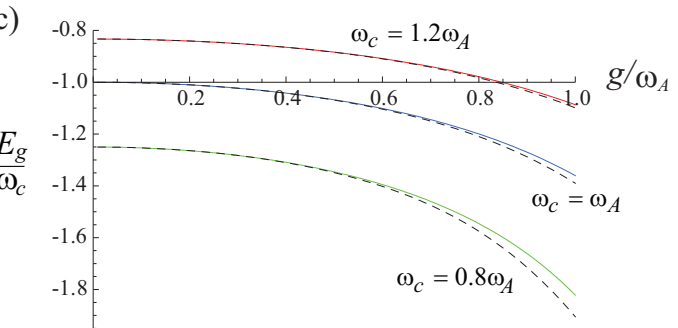

(d)

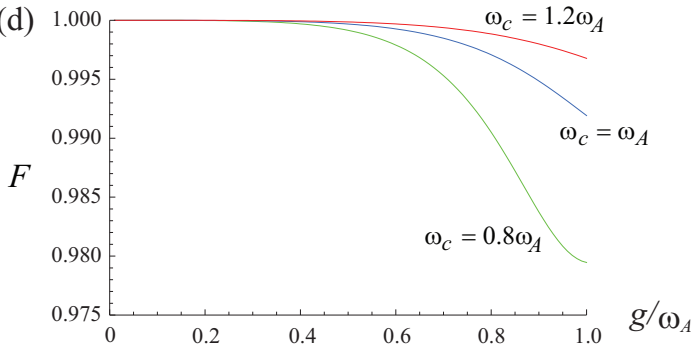

FIG. 1: (Color online) [(a)-(b)] Solution of the parameters $\alpha$ and $\beta$ that minimize $\langle H\rangle$ as a function of $g$ for various detunings. (c) The ground state energy as a function of coupling strength $g / \omega_{A}$. The solid line corresponds to $E_{v}$ from the variational method and the dashed line corresponds to the exact numerical values $E_{g}$. (d) The fidelity of the ground state obtained by the variational method.

i.e., $J_{z}|m\rangle_{A}=m|m\rangle_{A}$ with $m=0, \pm 1$. Then the ground state of the system can be expressed as $\left|\psi_{g}\right\rangle=$ $\sum_{m=0, \pm 1}\left|f_{m}\right\rangle_{F}|m\rangle_{A}$, with $\left|f_{m}\right\rangle_{F}$ being the field state associated with the atomic state $|m\rangle_{A}$.

To search for $\left|f_{m}\right\rangle$, we make use of variational method. Specifically, we assume that $\left|f_{m}\right\rangle$ are coherent states so that the trial ground state is in the form:

$$
\left|\psi_{v}\right\rangle=\frac{1}{N}\left(|\alpha\rangle_{F}|-1\rangle_{A}+\beta|0\rangle_{F}|0\rangle_{A}+|-\alpha\rangle_{F}|1\rangle_{A}\right) .
$$

where $|\alpha\rangle_{F}$ is a coherent state of the field with a real amplitude $\alpha, \beta$ is a variation parameter, and $N^{2}=2+\beta^{2}$ is the normalization constant. The expectation value of the Hamiltonian $\langle H\rangle$ with respect to $\left|\psi_{v}\right\rangle$ is given by,

$$
\langle H\rangle=\frac{2}{2+\beta^{2}}\left(\alpha^{2} \omega_{c}-2 \alpha g+\sqrt{2} \beta \omega_{A} e^{-\frac{\alpha^{2}}{2}}\right) .
$$

By varying the parameters $\alpha$ and $\beta,\langle H\rangle$ is minimized at $\frac{\partial\langle H\rangle}{\partial \alpha}=0$ and $\frac{\partial\langle H\rangle}{\partial \beta}=0$, which give the conditions:

$$
\begin{aligned}
& \beta=\frac{\sqrt{2}}{\alpha \omega_{A}}\left(\alpha \omega_{c}-g\right) e^{\frac{\alpha^{2}}{2}}, \\
& g=\frac{\alpha^{2} \beta \omega_{c}-\left(2-\beta^{2}\right) \frac{\omega_{A}}{\sqrt{2}} e^{-\frac{\alpha^{2}}{2}}}{2 \alpha \beta} .
\end{aligned}
$$

The values of $\alpha$ and $\beta$ can be obtained by solving the above equations numerically (Fig. 1a and b), and hence the approximate ground state energy $E_{v}=\langle H\rangle$ can be determined. We remark that there are two sets of $\alpha$ and $\beta$ which satisfy the conditions given in Eqs. (5) and (6), but only the set with $\alpha<g / \omega_{c}$ minimizes $\langle H\rangle$. In addition, if the coupling strength $g$ is smaller than $\omega_{c}+\omega_{A}$, then $\alpha$ and $\beta$ take the following approximate forms:

$$
\begin{aligned}
\alpha & \approx \frac{g}{\omega_{A}+\omega_{c}}, \\
\beta & \approx-\sqrt{2}+\frac{2 \omega_{A}+\omega_{c}}{\sqrt{2} \omega_{A}\left(\omega_{A}+\omega_{c}\right)^{2}} g^{2} .
\end{aligned}
$$

For the parameter used in Fig. 1, we see that Eqs. (7) and (8) can well capture the exact values of $\alpha$ and $\beta$ for small $g$.

We have tested the accuracy of the variational method by comparing the approximate ground state energy $E_{v}$ with the numerically exact eigenvalues $E_{g}$ near the resonance situation with $\omega_{A} \approx \omega_{c}$. The comparison is graphically shown in Fig. 1c. We see that the variational solution can produce the ground state energies very well agree with the exact values for $g / \omega_{A} \leq 0.5$. For example, the error is about $0.1 \%$ at $g=0.5 \omega_{A}$ at the exact resonance. In fact, the variational solution works in a wider range of $g$ when there is a positive detuning $\omega_{c}-\omega_{A}>0$. This is shown in the curves $\omega_{c}=1.2 \omega_{A}$ in which there is no visible discrepancy for $g / \omega_{A}<0.8$.

Another measure of the accuracy is the fidelity defined by inner product between the exact numerical ground state vector and the variational state vector, i.e., $F=$ $\left\langle\psi_{v} \mid \psi_{g}\right\rangle$. The results are shown in Fig. 1d, where we have $F>0.999$ for $g / \omega_{A}<0.5$ for the resonance case.

\section{TRANSFORMATION METHOD}

In this section, we describe an alternative method to obtain the ground state of the system. Such a method 
is based on a unitary transformation that simplifies the Hamiltonian to a form without countering rotating terms approximately. In the case of the (spin-half) Rabi model, the corresponding unitary transformation has been discussed in Refs. [16 18]. Here we indicate how the method can be extended to our spin-1 model.

To begin with we consider a unitary transformation defined by $H^{\prime}=e^{S} H e^{-S}$. Here

$$
S=\chi\left(a^{\dagger}-a\right) J_{z}
$$

with $\chi$ being a parameter to be determined. Under the unitary transformation, $H^{\prime}$ can be expressed as

$$
H^{\prime}=H_{0}^{\prime}+H_{1}^{\prime}+H_{2}^{\prime},
$$

with

$$
\begin{aligned}
H_{0}^{\prime} & =\eta \omega_{A} J_{x}-\left(2 g \chi-\omega_{c} \chi^{2}\right) J_{z}^{2}+\omega_{c} a^{\dagger} a \\
H_{1}^{\prime} & =\left(g-\omega_{c} \chi\right)\left(a^{\dagger}+a\right) J_{z}+i \eta \omega_{A} \chi\left(a^{\dagger}-a\right) J_{y} \\
H_{2}^{\prime} & =\omega_{A} J_{x}\left\{\cosh \left[\chi\left(a^{\dagger}-a\right)\right]-\eta\right\} \\
& +i \omega_{A} J_{y}\left\{\sinh \left[\chi\left(a^{\dagger}-a\right)\right]-\eta \chi\left(a^{\dagger}-a\right)\right\}
\end{aligned}
$$

Here $\eta=e^{-\frac{\chi^{2}}{2}}$ is defined. The $\cosh \left[\chi\left(a^{\dagger}-a\right)\right]$ and $\sinh \left[\chi\left(a^{\dagger}-a\right)\right]$ terms in $H_{2}^{\prime}$ has the leading expansion term:

$$
\begin{aligned}
& \cosh \left(\chi\left(a^{\dagger}-a\right)\right)=\eta+O\left(\chi^{2}\right), \\
& \sinh \left(\chi\left(a^{\dagger}-a\right)\right)=\eta \chi\left(a^{\dagger}-a\right)+O\left(\chi^{3}\right) .
\end{aligned}
$$

Therefore $H_{2}^{\prime}$ is of order $O\left(\chi^{2}\right)$ describing higher order (multi-photon) processes, and we shall neglect $H_{2}^{\prime}$ as an approximation, i.e., $H^{\prime} \approx H_{0}^{\prime}+H_{1}^{\prime}$. This is the same approximation procedure in the spin-half system [16 18]. However, the main difference here is the presence of the $J_{z}^{2}$ operator term in $H_{0}^{\prime}$. This is in contrast to the spinhalf system in which the corresponding $S_{z}^{2}$ term is just a constant.

To proceed, it is useful to express the atomic part of $H_{0}^{\prime}+H_{1}^{\prime}$ in the diagonal basis of $\eta \omega_{A} J_{x}-\left(2 g \chi-\omega_{c} \chi^{2}\right) J_{z}^{2}$. Such an operator appears in $H_{0}^{\prime}$ and it describes a renormalized three-level system. Specifically, we solve $\left[\eta \omega_{A} J_{x}-\left(2 g \chi-\omega_{c} \chi^{2}\right) J_{z}^{2}\right]|\nu\rangle=\varepsilon_{\nu}|\nu\rangle$ for eigenvectors $|\nu\rangle$ and eigenvalues $\varepsilon_{\nu}$, with the index $\nu=0, \pm$ labelling the eigenvectors such that $\varepsilon_{+}>\varepsilon_{0}>\varepsilon_{-}$. It can be shown that $\varepsilon_{0}=-\mu \eta \omega_{A}, \varepsilon_{ \pm}=\eta \omega_{A}\left(-\mu \pm \sqrt{4+\mu^{2}}\right) / 2$ with $\mu \equiv\left(2 g \chi-\omega_{c} \chi^{2}\right) / \eta \omega_{A}$, and the eigenvectors are:

$$
\begin{aligned}
& | \pm\rangle=\frac{1}{N_{ \pm}}\left(|1\rangle_{A}+\lambda_{ \pm}|0\rangle_{A}+|-1\rangle_{A}\right), \\
& |0\rangle=\frac{1}{\sqrt{2}}\left(|1\rangle_{A}-|-1\rangle_{A}\right),
\end{aligned}
$$

where $\lambda_{ \pm}=\left(\mu \pm \sqrt{4+\mu^{2}}\right) / \sqrt{2}$ and $N_{ \pm}^{2}=2+\lambda_{ \pm}^{2}$ are the normalization constants. In this way,

$$
H^{\prime} \approx \omega_{c} a^{\dagger} a I_{A}+\left(\begin{array}{ccc}
\varepsilon_{-} & c_{1} a+c_{2} a^{\dagger} & 0 \\
c_{2} a+c_{1} a^{\dagger} & \varepsilon_{0} & c_{3} a+c_{4} a^{\dagger} \\
0 & c_{4} a+c_{3} a^{\dagger} & \varepsilon_{+}
\end{array}\right),
$$

where $I_{A}$ is the $3 \times 3$ identity matrix operating on the atomic subspace. The $c_{j}$ are some constants depending on the parameter $\chi$. We see in Eq. (18) that the terms involving coefficients $c_{1}$ and $c_{3}$ are counter rotating, because they describe virtual processes in which atomic excitation is accompanied by the emission of a photon.

The main purpose of transforming $H$ into $H^{\prime}$ is to eliminate some of the counter rotating terms by using a suitable parameter $\chi$. Unlike the spin-half atom, it is not possible to find a $\chi$ to remove all counter rotating terms. However, since we are interested in the ground state, it is sufficient to find $\chi$ that makes $c_{1}=0$, because in this case the corresponding $H^{\prime}$ in Eq. (18) has the eigenvector $|0\rangle_{F}|-\rangle_{A}$, which is expected to be the ground state vector if the interaction is not too strong. Returning to the original frame, we have the approximation for the ground state energy:

$$
E_{g} \approx \varepsilon_{-}=-\frac{\eta \omega_{A}\left(\mu+\sqrt{4+\mu^{2}}\right)}{2},
$$

and the ground state vector $\left|\psi_{g}\right\rangle \approx\left|\psi_{T}\right\rangle$ (subscript $T$ for the transformation method), where

$$
\begin{aligned}
\left|\psi_{T}\right\rangle & =e^{-S}|0\rangle_{F}|-\rangle_{A} \\
& =\frac{1}{N_{-}}\left(|\chi\rangle_{F}|-1\rangle_{A}+\lambda_{-}|0\rangle_{F}|0\rangle_{A}+|-\chi\rangle_{F}|1\rangle_{A}\right) .
\end{aligned}
$$

Here $|\chi\rangle_{F}$ denotes the coherent state of the field with the amplitude $\chi$. We see that this is exactly the same form of the trial variational function given in Eq. (3). The value of $\chi$ is determined by the condition $c_{1}=0$ which gives,

$$
\lambda_{-}=\frac{\sqrt{2}}{\chi \omega_{A}}\left(\chi \omega_{c}-g\right) e^{\frac{\chi^{2}}{2}} .
$$

Moreover, by $\lambda_{-}=\frac{\mu-\sqrt{4+\mu^{2}}}{\sqrt{2}}$ and $\mu=\frac{2 g \chi-\omega_{c} \chi^{2}}{\eta \omega_{A}}$, we have

$$
g=\frac{\chi^{2} \lambda_{-} \omega_{c}-\left(2-\lambda_{-}^{2}\right) \frac{\omega_{A}}{\sqrt{2}} e^{-\frac{\chi^{2}}{2}}}{2 \chi \lambda_{-}} .
$$

Comparing Eqs. (21) and (22) with Eqs. (5) and (6) obtained in the variational method, we can see that the transformation method and the variational method give the same ground state, with $\chi$ and $\lambda_{-}$are equivalent to $\alpha$ and $\beta$ respectively, i.e., we have $\varepsilon_{-}=E_{v},\left|\psi_{T}\right\rangle=\left|\psi_{v}\right\rangle$. Therefore the transformation procedure in this section can be understood as a variation approach, in the sense that we vary the Hamiltonian by the unitary transformation, instead of the trial wave function.

However, the main advantage of the transformation method is that the corrections can be identified explicitly. This is because $H_{2}^{\prime} \approx 0$ is the only approximation made in the above derivation. Therefore the exact ground state involves higher order processes described by $H_{2}^{\prime}$, and this information cannot be obtained by the simply varying 
the wave function as in Sec. II. Specifically, for $\chi<1$, the leading contribution of $H_{2}^{\prime}$ is

$$
H_{2}^{\prime} \approx \frac{\eta \chi^{2} \omega_{A}}{2} J_{x}\left(a^{\dagger 2}-2 a^{\dagger} a+a^{2}\right),
$$

which comes from the expansion of the $\cosh \left[\chi\left(a^{\dagger}-a\right)\right]$ term. By treating Eq. (23) as a weak perturbation, we can use the perturbation theory to find the correction of the ground state energy (up to second order of $H_{2}^{\prime}$ ):

$$
\begin{aligned}
& E_{g} \approx \varepsilon_{-}+\delta E \\
& \delta E=-\frac{2 \chi^{4}}{N_{-}^{2}}\left[\frac{2 \varepsilon_{+}^{2}}{N_{-}^{2} \omega_{c}}+\frac{\varepsilon_{0}^{2}}{N_{+}^{2}\left(2 \omega_{c}+\varepsilon_{+}-\varepsilon_{-}\right)}\right] .
\end{aligned}
$$

In Table I, we indicate the performance of the approximation scheme for the resonance case $\omega_{A}=\omega_{c}$. We see that although $\varepsilon_{-}$is already quite close to the exact eigenvalue $E_{g}$, there is a significant improvement by including the correction $\delta E$, by comparing the second and forth columns in the table.

\begin{tabular}{crrc}
\hline \hline$g / \omega_{A}$ & $E_{g} / \omega_{A}$ & $\varepsilon_{-} / \omega_{A}$ & $\left(\varepsilon_{-}+\delta E\right) / \omega_{A}$ \\
\hline 0.2 & -1.01015 & -1.01013 & -1.01015 \\
0.4 & -1.04256 & -1.04210 & -1.04255 \\
0.6 & -1.10404 & -1.10137 & -1.10403 \\
0.8 & -1.20984 & -1.19965 & -1.20988 \\
1.0 & -1.38986 & -1.36052 & -1.39094 \\
1.2 & -1.68602 & -1.62699 & -1.68995 \\
\hline \hline
\end{tabular}

TABLE I: A list of ground state energy obtained by various methods at $\omega_{c}=\omega_{A} . E_{g}, \varepsilon_{-}$, and $\left(\varepsilon_{-}+\delta E\right) / \omega_{A}$ are defined in the text.

\section{QUANTUM ENTANGLEMENT BETWEEN QUBITS}

In this section we examine the quantum entanglement between the two qubits when the system is in the ground state. Negativity is employed in order to quantify how the two qubits are entangled in the ultrastrong coupling regime. The negativity is defined as [27]

$$
\mathcal{N}\left(\rho_{A}\right) \equiv \frac{\left\|\rho_{A}^{T_{A}}\right\|_{1}-1}{2},
$$

where $\left\|\rho_{A}^{T_{A}}\right\|_{1}$ is the trace norm of $\rho_{A}^{T_{A}}, \rho_{A}^{T_{A}}$ is the partial transpose of the reduced density matrix $\rho_{A}=\operatorname{Tr}_{F}(\rho)$ and $\rho=\left|\psi_{g}\right\rangle\left\langle\psi_{g}\right|$. Alternatively, Eq. (26) can be calculated by the absolute value of the sum of the negative eigenvalues of $\rho_{A}^{T_{A}}$.

Using the approximate ground state vector $\left|\psi_{v}\right\rangle$ in Eq. (3), the corresponding reduced density matrix $\rho_{A}$ can be expressed as

$$
\rho_{A}=\frac{1}{2 N^{2}}\left(\begin{array}{cccc}
\rho_{11} & 0 & 0 & \rho_{14} \\
0 & \rho_{22} & \rho_{23} & 0 \\
0 & \rho_{32} & \rho_{33} & 0 \\
\rho_{41} & 0 & 0 & \rho_{44}
\end{array}\right),
$$

where

$$
\begin{aligned}
& \rho_{11}=1+\beta^{2}+2 \sqrt{2} \beta e^{-\frac{\alpha^{2}}{2}}+e^{-2 \alpha^{2}}, \\
& \rho_{14}=\rho_{41}=1-\beta^{2}+e^{-2 \alpha^{2}}, \\
& \rho_{22}=\rho_{23}=\rho_{32}=\rho_{33}=1-e^{-2 \alpha^{2}}, \\
& \rho_{44}=1+\beta^{2}-2 \sqrt{2} \beta e^{-\frac{\alpha^{2}}{2}}+e^{-2 \alpha^{2}},
\end{aligned}
$$

and the order of the columns and rows is $|e e\rangle,|e g\rangle,|g e\rangle$ and $|g g\rangle$ which correspond to the bare atomic level of the two qubits. The four eigenvalues of $\rho_{A}^{T_{A}}$ can be obtained analytically and only one of them is negative. Thus, the expression of the approximate negativity $\mathcal{N}$ is

$$
\mathcal{N}=\max \left\{\frac{2 e^{-2 \alpha^{2}}-\beta^{2}}{2\left(2+\beta^{2}\right)}, 0\right\}
$$

In the limit $g / \omega_{A} \ll 1, \alpha$ and $\beta$ are approximated by Eqs. (7) and (8), and this gives

$$
\mathcal{N} \approx \frac{\omega_{c}}{4 \omega_{A}\left(\omega_{A}+\omega_{c}\right)^{2}} g^{2} .
$$

Therefore the degree of entanglement increases with $g^{2}$ when $g / \omega_{A} \ll 1$. As a remark, we have also calculated the corresponding concurrence and it is simply twice of $\mathcal{N}$ calculated in the Eq. (32).

We have computed the negativity from the numerically exact ground state for the resonance case, and it is plotted in Fig. 2 with a dashed line. We see that the negativity is well approximated by Eq. (32) (solid line) for $g / \omega_{A}<0.5$. In particular, the quadratic dependence of $g$ is captured by Eq. (33) at small $g$. There is a greater discrepancy when $g / \omega_{A}>0.5$, which is consistent with fidelity $F$ behavior shown in Fig. 1d. Although Fig. 2 does not show the case of $g / \omega_{A}>1$, the exact negativity drops as $g / \omega_{A}$ further increases after it reaches its maximum at $g \approx \omega_{A}$. When $g \approx 2.6 \omega_{A}$, the negativity would drop to zero and never increase again. In other words, the two qubits become disentangled if $g$ is sufficiently large.

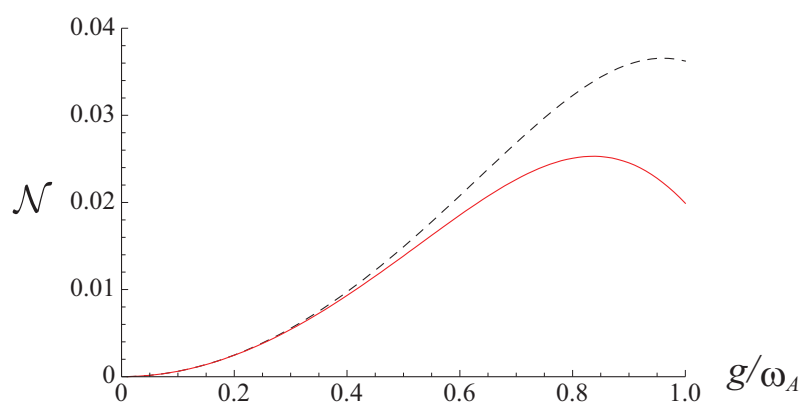

FIG. 2: (Color online) The negativity $\mathcal{N}$ as a function of coupling strength $g / \omega_{A}$ for the $\omega_{c}=\omega_{A}$ case. The solid line (dashed line) corresponds to the negativity of the approximate (exact) ground state. 


\section{CONCLUSION}

To conclude, we have shown that the ground state of the Rabi model formed by two identical qubits in the near resonance configuration can be determined approximately by using a variational method and a transformation method, and the results are in good agreement with exact numerical calculations when $g$ is a significant fraction of $\omega_{A}$ (up to $g / \omega_{A}=0.5$ ). For instance, the error of the variational ground state energy for the exact resonance case is about $0.1 \%$ at $g=0.5 \omega_{A}$, and the error can be greatly reduced to $0.0004 \%$ by further using second order perturbation theory. The key advantage of our methods is that the analytical form of the ground state can be captured approximately, which is useful for analyzing the ground state properties. Specifically, we have examined the quantum entanglement between the two qubits. Such an entanglement is a consequence of counter rotating terms in the Hamiltonian, because if such terms are dropped by RWA, then the two qubits are simply disentangled in the ground state. Using our approximate ground state vector, we are able to determine the negativity as a function of system parameters for $g / \omega_{A}$ up to 0.5 .

\section{Acknowledgments}

This work is partially supported by a grant from the Research Grants Council of Hong Kong, Special Administrative Region of China (Project No. CUHK401812).
[1] T. Niemczyk, F. Deppe, H. Huebl, E. P. Menzel, F. Hocke, M. J. Schwarz, J. J. Garcia-Ripoll, D. Zueco, T. Hümmer, E. Solano, A. Marx, and R. Gross, Nat. Phys. 6, 772 (2010).

[2] P. Forn-Díaz, J. Lisenfeld, D. Marcos, J. J. García-Ripoll, E. Solano, C. J. P. M. Harmans, and J. E. Mooij, Phys. Rev. Lett. 105, 237001 (2010).

[3] G. Günter, A. A. Anappara, J. Hees, A. Sell, G. Biasiol, L. Sorba, S. De Liberato, C. Ciuti, A. Tredicucci, A. Leitenstorfer, and R. Huber, Nature 458, 178(2009).

[4] Y. Todorov, A. M. Andrews, R. Colombelli, S. De Liberato, C. Ciuti, P. Klang, G. Strasser, and C. Sirtori, Phys. Rev. Lett. 105, 196402 (2010).

[5] X. Cao, J. Q. You, H. Zheng, and F. Nori, New. J. Phys. 13, 073002 (2011).

[6] A. Ridolfo, M. Leib, S. Savasta, and M. J. Hartmann, Phys. Rev. Lett. 109, 193602 (2012).

[7] S. Ashhab and F. Nori, Phys. Rev. A 81, 042311 (2010).

[8] S. Ashhab, Phys. Rev. A 87, 013826 (2013).

[9] J. Casanova, G. Romero, I. Lizuain, J. J. García-Ripoll, and E. Solano, Phys. Rev. Lett. 105, 263603 (2010).

[10] I. I. Rabi, Phys. Rev. 49, 324 (1936); 51, 652 (1937).

[11] E. A. Tur, Opt. Spectrosc. 89, 574 (2000).

[12] D. Braak, Phys. Rev. Lett. 107, 100401 (2011).

[13] Joachim Stolze and Lothar Müller, Phys. Rev. B 42, 6704 (1990).

[14] Hong Chen, Yu-Mei Zhang, and Xiang Wu, Phys. Rev.
B 40, 11326 (1989).

[15] E. K. Irish, Phys. Rev. Lett. 99, 173601 (2007).

[16] C. J. Gan and H. Zheng, Eur. Phys. J. D 59, 473 (2010).

[17] Zhiguo Lü and Hang Zheng, Phys. Rev. B 75, 054302 (2007).

[18] Xiufeng Cao, J. Q. You, H. Zheng, A. G. Kofman, and Franco Nori, Phys. Rev. A 82, 022119 (2010).

[19] Lixian Yu, Shiqun Zhu, Qifeng Liang, Gang Chen, and Suotang Jia, Phys. Rev. A 86, 015803 (2012).

[20] T. Liu, K. L. Wang, and M. Feng, Europhys. Lett. 86, 54003 (2009).

[21] J. Hausinger and M. Grifoni, Phys. Rev. A 82, 062320 (2010).

[22] Myung-Joong Hwang and Mahn-Soo Choi, Phys. Rev. A 82, 025802 (2010).

[23] V. V. Albert, G. D. Scholes, and P. Brumer, Phys. Rev. A 84, 042110 (2011).

[24] Y. Zhang, G. Chen, L. Yu, Q. Liang, J.-Q. Liang, and S. Jia, Phys. Rev. A 83, 065802 (2011).

[25] M. Tavis and F. W. Cummings, Phys. Rev. 170, 379 (1968); M. Tavis and F. W. Cummings, Phys. Rev. 188, 692 (1969).

[26] S. Agarwal, S. M. Hashemi Rafsanjani, and J. H. Eberly, Phys. Rev. A 85, 043815 (2012).

[27] G. Vidal, R. F. Werner, Phys. Rev. A 65, 032314 (2002). 